\title{
CS Research Square

\section{Transcriptome profilings reveal the candidate genes, pathways and transcription factors related to nitrogen utilization and excessive nitrogen stress in perennial ryegrass.}

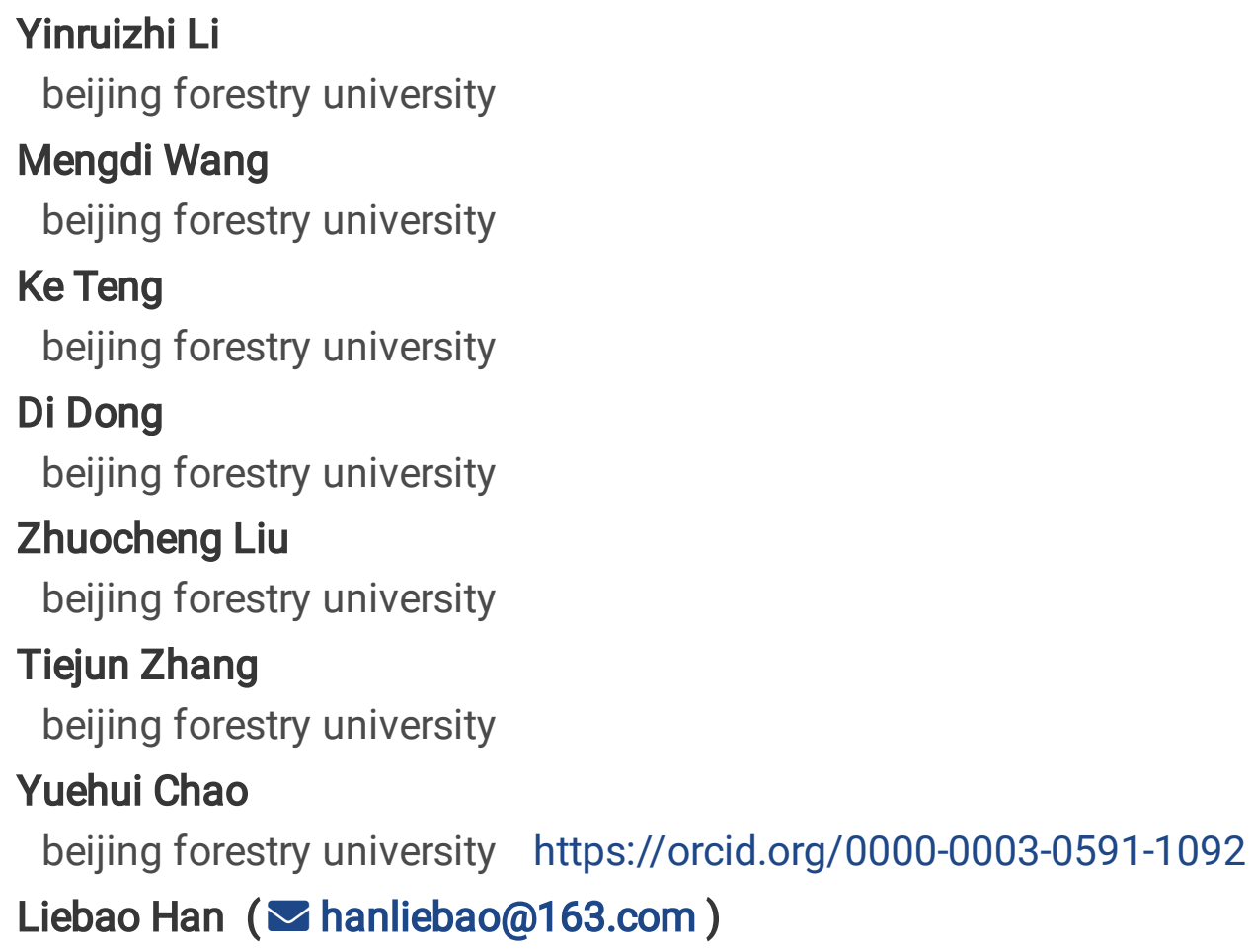

Research article

Keywords: Perennial ryegrass, transcriptome, nitrogen utilization, excessive nitrogen stress.

Posted Date: May 1st, 2020

DOI: https://doi.org/10.21203/rs.3.rs-25633/v1

License: (c) (1) This work is licensed under a Creative Commons Attribution 4.0 International License. Read Full License 


\section{Abstract}

Background $\mathbb{L}$ Lolium perenne L. is a kind of high quality forage grass, which can provide a good nutritional basis for herbivorous livestock. However, how to improve the nitrogen utilization rate of ryegrass and avoid the nitrate toxicity caused by excessive nitrogen has been troubling people for a long time. Up to now, the molecular response mechanism of ryegrass to nitrogen is not clear, especially under the condition of excessive nitrogen. Based on this, we tried to obtain a new insight into molecular response of ryegrass in nitrogen utilization and excessive nitrogen stress, providing the molecular theoretical basis for solving this problem.

Results: In this study, the transcription of perennial ryegrass at different nitrogen levels was identified by high-throughput next-generation DNA sequencing. Phenotypic characterizations investigated that ryegrass in treatment N0.5 has a better growth state than the other three groups. The treatment N1 and N10 contained excessive nitrogen, which had a stress effect on plant growth. Analysis of differentially expressed genes indicated that 345, 105 genes are considered to involve in the regulation of nitrogen utilization and excessive nitrogen stress, respectively. GO enrichment analysis revealed that plant response to nitrogen mainly enrich in two categories, including "biological process" and "molecular function". KEGG enrichment analysis suggested that "Photosynthesis-antenna proteins" may respond positively to nitrogen under appropriate nitrogen conditions, whereas "steroid biosynthesis", "carotenoid biosynthesis" and "C5-branched dibasic acid metabolism" had been identified as top significant enrichment pathways response to excessive nitrogen. Transcription factors analysis showed that 21 TFs related to nitrogen utilization were classified into 10 transcription factor families, especially AP2-EREBP and MYB TF families. 4 TFs related to excessive nitrogen stress were identified, which belonged to 4 transcription factor families including LOB, NAC, AP2-EREBP and HB. The expression patterns of these selected genes above were also analyzed.

Conclusions: These results made a contribution to comprehend the molecular mechanism of perennial ryegrass response to nitrogen. It provides new ideas for guiding the production practice and variety improvement of forage and even food crops from the perspective of molecular biology.

\section{Background}

Nitrogen is the main nutrient element for plant growth and plays a very important role in promoting crop yield increase. Appropriately increasing the application of nitrogen fertilizer is one of the necessary measures to achieve high yield. However, excessive application of nitrogen fertilizer not only reduces the Nitrogen utilization and utilization efficiency of crops, but also causes a series of problems such as reduced crop planting efficiency, resource waste and environmental pollution(Bijay-Singh, et al. 1995). It is of great significance to study the reasonable application rate of nitrogen fertilizer for the sustainable development of agriculture, which can save cost, increase benefit and reduce environmental pollution. With the development of molecular biology, many genes related to nitrogen utilization have been cloned and identified, which helps to further elucidate the molecular mechanism of nitrogen uptake and 
utilization in crops, and lays a foundation for improving nitrogen utilization efficiency in crops by means of genetic engineering.

The processes that occur when nitrogen enters plants include absorption, transport and distribution. There are several genes in plants that regulate nitrogen uptake and it was reported that two transporter gene families have been involved in root nitrogen uptake in plants: nitrate transporter 1/ small peptide transporter family NPF (NRT1/PTR) and $\mathrm{NO}_{3}{ }^{-}$transporter family (NRT2). The NRT1 and NRT2 families in Arabidopsis Thaliana had 53 and 7 genes respectively. NRT1 was a subset of low affinity $\mathrm{NO}_{3}{ }^{-}$transfer system (LATS), and NRT2 was a component of high affinity NO3- transfer system (HATS)(Kotur and GLASS 2015). The absorption mechanism of $\mathrm{NH}_{4}{ }^{+}$by plant roots was similar to the absorption mechanism of $\mathrm{NO}_{3}{ }^{-}$, and also has HATS and LATS absorption and transport systems. Among them, the high-affinity absorption and transport system (HATS) of $\mathrm{NH}_{4}{ }^{+}$depended on the AMT gene family from the root epidermal cells and membrane of the cortex, which are divided into AMT1 and AMT2 subfamilies(Wang, et al. 2018). In addition, four $\mathrm{NO}_{3}{ }^{-}$transport families had been found to be involved in the transport, distribution and utilization of $\mathrm{NO}_{3}{ }^{-}$in plants: NPF (NRT1/pTR)Family, NRT2 family, chloride channel family (CLC), s-type anion channels and their homologues (SLAC/SLAH)(Wang, et al.). At present, the increasing advances have been made in principles of the physiology and molecular biology about nitrogen uptake, transport, distribution and their regulation in plants. However, the current researches mainly focus on some bulk plants whose performance is greatly affected by the environment, while there are few researches on molecular mechanism of plant response to nitrogen in other species with relatively narrow application ranges.

Perennial ryegrass (Lolium perenne L.) is a kind of high quality forage grass, which can provide a good nutritional basis for herbivorous livestock and promote the development of animal husbandry(Byrne, et al. 2015). However, as a grass family, perennial ryegrass itself does not have the ability to fix nitrogen, and nitrogen for its growth and development comes mainly from the absorption of root systems from the soil. Nitrogen available in the soil is often difficult to meet the needs of forage grass. Therefore, supplementing soil nitrogen with fertilizer is one of the most effective measures for high quality and high yield of forage. It should be noted that more artificial nitrogen fertilizer does not mean the more nitrogen plant can absorb. When the nitrogen application exceeds a certain level, the utilization efficiency of nitrogen will decrease and the nitrate will be enriched in plants, leading to poisoning of grazing livestock by feeding on those with high nitrate content(Wright and J. 1964). Therefore, it is of great importance to study the endogenous molecular mechanism of Nitrogen utilization and excessive nitrogen stress in perennial ryegrass, which can provide a theoretical foundation for guiding production practice.

In recent years, studies about nitrogen in ryegrass have focused on its effect on the yield and quality of forage grass, livestock and agricultural by-products. A mini-sward study in Valdivia found the lower the frequency of nitrogen application, the higher the total crude protein content of ryegrass, but the crude protein component was not affected by the application frequency(Loaiza P 2019). Another study showed that applying high levels of nitrogen to a tetraploid or diploid perennial ryegrass meadow, mixed with 
white clover, effectively increased milk production per hectare per cow(McClearn, et al. 2019). In addition, the molecular response mechanism of nitrogen in ryegrass is rarely reported.

In this study, we used high-throughput next-generation DNA sequencing (NGS) technologies to identify the transcription of perennial ryegrass at different nitrogen levels, in order to screen candidate genes related to Nitrogen utilization and stress and to further understand the molecular mechanism related to nitrogen in ryegrass. Several transcriptomes related to nitrogen in plant have been characterized respectively in rice(Huang, et al. 2016), potato(Tiwari, et al. 2020), arabidopsis(Peng, et al. 2007), wheat(Ruuska, et al. 2008), sorghum(Gelli, et al. 2017), poplar(Lu, et al. 2019), nankingense(Wang, et al. 2015), tea(Yang, et al. 2018). However, some of these studies focused on carbon and nitrogen balance at the transcriptional level and the other part focused on the change of transcriptional level under the condition of nitrogen deficiency. On the basis of previous studies, our study explored changes in the transcription level of ryegrass under the condition of excessive nitrogen, which compensated for the molecular mechanism of plant response to nitrogen to some extent.

\section{Results}

\section{Phenotypic characterization of perennial ryegrass at different concentration of nitrogen}

To describe the growth state of perennial ryegrass under different treatments(Fig. 1), plant height, root length, fresh weight, tiller number and chlorophyll content all samples were determined(Fig. 2A-E). The results showed that plant height of treatment $\mathrm{N} 0.5$ and $\mathrm{N} 1$ was significantly higher than the two others(Fig. 2A). The fresh weight and tiller number of N0.5 treatment were significantly higher than those of other treatments(Fig. 2C,2D). However, the root length of treatment NO as control was highest and significantly higher than that in other treatments(Fig. 2B). The results of chlorophyll content determination indicated that there is a significant ascension in treatment N0.5 compared to the other three groups, meanwhile, a trend of first up and then down was verified among these four treatments(Fig. 3). Based on the above, it could be inferred that ryegrass in treatment N0.5 has a better growth state than the other three groups. The treatment N1 and N10 contained excessive nitrogen, which had a stress effect on plant growth.

\section{Sequencing and transcriptome assembly}

To relatively comprehensive identified the transcripts of perennial ryegrass response to nitrogen, there were 12 cDNA libraries constructed from four treatments(N0,N0.5,N1,N10), orderly corresponding to the conditions of no nitrogen, moderate amounts of nitrogen, a certain amount of excessive nitrogen and high concentration excessive nitrogen, each of which had three biological replications. Totally, $80.58 \mathrm{~GB}$ clean data were released, which the content of each sample varied from $6.02 \mathrm{~GB}$ to $7.20 \mathrm{~GB}$ (table S1). All these clean data were performed genomic localization analysis(http://185.45.23.197:5080/ryegrassgenome) by HISAT. A minimum of total mapped clean data 
occupied $61.84 \%$ in the relative example and the proportion of multiple mapped reads or fragments in each example was less than $0.8 \%$ (table S2). Through transcriptome assembly, these transcripts were classified into 101377 unigenes, among which 58732 unigenes were successfully annotated.

\section{Analysis of differentially expressed genes(DEGs)}

In order to understand the response of ryegrass gene to nitrogen, DEGs in three treatments(N0.5,N1 and $\mathrm{N} 10$ ) were screened with treatment $\mathrm{N} 0$ as control. The screening criteria were as follows: FoldChange $>2$, $-\log _{10}($ pvalue $)>1$.3. Under these conditions, $883,1597,1778$ were identified separately as significantly differentially expressed in the treatment N0.5, N1 and N10. In treatment N0.5, there were 422 genes upregulated and 461 genes down-regulated, compared to the control group. Accordingly, the expression of 546 genes was up-regulated and the expression of 1051 genes was down-regulated in treatment N1. 642 genes were up-regulated and 1166 genes were down-regulated in treatment N10(Fig. 4A-C).

The differential expression of these DEGs was caused by the addition of nitrogen. In order to find out more about genes related specifically to Nitrogen utilization, three groups of DEGs were drawn into a Venn diagram(Fig. 5a). The results showed that 345 genes are considered to involve in the regulation of Nitrogen utilization directly, among which 322 genes are known and the rest are novel genes.

In plant growth status identification, it could be found that conditions of treatment N0.5 are more suitable for ryegrass growth than the other three groups, and treatment N1 and N10 produces excessive nitrogen stress on the plants. Based on this, we further attempted to screen genes that specifically respond to excessive nitrogen stress. DEGs in treatment $\mathrm{N} 1$ and $\mathrm{N} 10$ were identified using treatment $\mathrm{N} 0.5$ as a control(Fig. 4D-E). It indicated that there are 456 differentially expressed genes(169 up-regulated, 287 down-regulated) and 494 differentially expressed genes(154 up-regulated, 340 down-regulated) in treatment $\mathrm{N} 1$ and $\mathrm{N} 10$. Further, 82 known genes and 22 novel genes were identified in the common genes in treatment $\mathrm{N} 1$ and $\mathrm{N} 10$ compared to treatment N0.5 (Fig. 5b). These common genes may play an important role in the response of perennial ryegrass to excessive nitrogen stress.

\section{Clustering of unigenes related to nitrogen utilization and excessive nitrogen stress}

To gain insights into the expression pattern of those screened unigenes related to Nitrogen utilization and excessive nitrogen stress, the expressions of them in the different treatments, containing different concentrations of nitrogen, were clustered separately(Fig. 6). Clustering analysis of unigenes related to Nitrogen utilization revealed that the expressions of those genes in treatment N0.5 was most similar to those in treatment $\mathrm{N} 1$, followed by those in treatment $\mathrm{N} 0$, and then those in treatment $\mathrm{N} 10$, in general(Fig. 6A). In consideration of the nitrogen content in different treatments, presumably we could tell that these genes have analogous expression in response to moderate amounts of nitrogen and a certain amount of excessive nitrogen, but distinct ones in response to high concentration excessive nitrogen. It was worth mentioning that the most part of genes related to Nitrogen utilization have the relatively high 
expressions in treatment $\mathrm{N} 10$, whereas they have the relatively low expressions in other three groups. It suggested that the most part of them possess similar expression pattern.

On the other hand, there were similar expressions of the genes related to nitrogen excessive nitrogen stress between treatment N0.5 and treatment N0, and something similar happened in treatment N1 and treatment N10(Fig. 6B). It could be inferred that those genes related to nitrogen excessive nitrogen stress express homoplastically under the condition of no nitrogen and moderate amounts of nitrogen, in view of there are different nitrogen content in each treatment. Similarly, these genes had analogous expression in response to excessive nitrogen ranging from a certain concentration to high concentration.

\section{GO and KEGG enrichment analysis of DEGs}

In order to further understand the functional classification and metabolic pathways involved in the response of perennial ryegrass to nitrogen, GO and KEGG enrichment of DEGs was analyzed. We selected $30 \mathrm{GO}$ terms with the most significant enrichment and showed them in the histogram(Fig. 7). GO enrichment analysis of DEGs in treatment N0.5 compared to treatment NO as control investigated that all DEGs selected have significantly enriched GO terms, which are classified into "biological process" and "molecular function", respectively. And "oxidation-reduction process" had maximum enrichment in all "biological process". The number of DEGs enriched in "catalytic activity", "transferase activity", "oxidoreductase activity" was also higher than the others in "molecular functior"(Fig. 7A). It suggested that the genes of plant response to nitrogen mainly enrich in "oxidation-reduction process", "catalytic activity", "transferase activity" and "oxidoreductase activity" under the conditions of relatively suitable nitrogen. Nevertheless, DEGs in treatment N1 compared to treatment N0.5 as control only had five significantly enriched GO terms, which were mainly classified into "molecular function" (Fig. 7B). We could deduce that more GO terms in "molecular function" are involved in the response mechanism of plants to nitrogen under a certain amount of nitrogen stress. Based on the $\mathrm{GO}$ enrichment analysis of DEGs in treatment N10 compared to treatment N0.5(Fig. 7C), there were no significantly enriched GO terms.

Furthermore, all DEGs were separately enriched in 90 pathways in total. 20 pathway items with the most significant enrichment were selected for display in the Fig. 8. DEG(ref0033532-processed-gene0.0 losa:4328052) in treatment N0.5 and N1(Fig. 6A,6B) displayed a certain degree of enrichment in the "nitrogen metabolism" pathway. The results in treatment N0.5 compared to NO showed that the top enriched pathway of DEGs is "Photosynthesis-antenna proteins", except "nitrogen metabolism"(Fig. 8A). It suggested that "Photosynthesis-antenna proteins" may also respond positively to nitrogen under appropriate nitrogen conditions. In the same way, "steroid biosynthesis" and "carotenoid biosynthesis" had been identified as top two significant enrichment pathways in the KEGG enrichment analysis of DEGs between treatment $\mathrm{N} 1$ and N0.5(Fig. 8B). In the meantime, there were two relatively enriched pathways("phenylpropanoid biosynthesis", "phenylalanine metabolism") with lower Qvalue(Fig. 8B). It mean that genes in these special pathways play an important role in the response mechanism of plant to a certain amount of nitrogen stress. About the pathways enriched in treatment N10 compared to N0.5, "C5-branched dibasic acid metabolism"(only Novel00490|osa:4328147 enriched) and "steriod 
biosynthesis" pathways showed the top significant enrichment(Fig. 8C). It can be indicated that "steriod biosynthesis" may perform some unknown and irreplaceable function under excessive nitrogen stress.

\section{Photosynthesis-antenna proteins, steroid biosynthesis and carotenoid biosynthesis pathways}

In KEGG enrichment analysis of DEGs, four special pathways with significantly enriched DEGs were found in three DEG groups caused by the different nitrogen content. In order to further explore the response of these pathways to different nitrogen conditions, the enriched DEGs were mapped to those filtrated pathways and the expressions of them were analyzed by clustering severally(Fig. 9). There were 9, 2, 2, 3 DEGs with significant enrichment classified into photosynthesis-antenna proteins, steroid biosynthesis and carotenoid biosynthesis pathways. These DEGs enriched in three special pathways displayed relatively broad expression patterns. Notably, the different DEGs in treatment N1 and N10 compared to treatment N0.5 were significantly enriched in steroid biosynthesis pathway simultaneously. It could be indicated that the diverse parts of steroid biosynthesis pathway function in perennial ryegrass response to excessive nitrogen ranging from a certain concentration to high concentration.

\section{Identification of transcription factors}

There is accumulating evidence that transcription factors (TFs) play key roles in various regulatory mechanisms(Hu, et al. 2017). Therefore, transcription factors from DEGs were identified for purpose of further studying the molecular regulatory network of perennial ryegrass in response to nitrogen(Fig. 10A). Totally, 185 TFs, among which there were 14 novel genes, were distinguished in all differentially expressed genes. These TFs belonged to 37 transcription factor families, including the top three TF families(AP2-EREBP, MYB, NAC) with quantities greater than ten.

The expression patterns of these TFs changed in a relatively large range. Based on this, TFs related to Nitrogen utilization and excessive nitrogen stress were characterized ulteriorly(Fig. 10B,10C). In total, 21 TFs related to Nitrogen utilization, among which AP2-EREBP and MYB TF families occupied the largest number, were classified into 10 transcription factor families(Fig. 10B). These TFs performed semblable expressions in treatment $\mathrm{N} 0.5, \mathrm{~N} 1$ and $\mathrm{N} 10$ and most of them possessed similar expression patterns in all treatments. Accordingly, 4 TFs related to excessive nitrogen stress were identified, which belonged to 4 transcription factor families including LOB, NAC, AP2-EREBP and HB(Fig. 10C). The expressions of these TFs were relatively alike in treatment $\mathrm{N} 1$ and N10, and 2 TFs in HB transcription factor family had the similar expression patterns. Remarkably, ref0030344-processed-gene-0.2 from AP2-EREBP transcription factor family performed positive response to nitrogen absorption and excessive nitrogen stress, concurrently.

\section{Expression validation by qRT-PCR}

To verify the accuracy of expressions from RNA-Seq, 22 unigenes with significantly different expressions were randomly selected for qRT-PCR analysis. These unigenes, among which 12 genes from DEGs related 
to nitrogen utilization and 10 from excessive nitrogen stress, contained 8 transcription factors and 14 functional genes. Almost all expression patterns of these genes were accordant in qRT-PCR analysis compared to those in the transcriptome profiling(Fig. 11). Thus, the results of qRT-PCR with good coherence to those of RNA-Seq contributed to reflect the High credibility and accuracy of transcriptome data.

\section{Discussion}

The response of plants to nitrogen as a key nutrient element for plant growth and development, performs a complex regulatory network involving in various kinds of functional genes and transcription factors. Illuminating these intricate molecular regulatory mechanisms would make great contributions to more scientific nitrogen application in the production practice. In this study, to produce added insight into the mechanisms of perennial ryegrass response to nitrogen, we paid more attention to those induced by Nitrogen utilization and excessive nitrogen stress. The transcripts of perennial ryegrass cultivated at different concentration of nitrogen were analyzed by RNA-Seq, mainly for identification of differentially expressed genes related to nitrogen utilization and excessive nitrogen stress.

\section{Phenotypic characterization of perennial ryegrass}

Under the condition of moderate amounts of nitrogen, the plants put up relatively larger plant height, fresh weight and tiller number compared to those under other conditions(Fig. 2A,C,D). On the other hand, it has been suggested, in some degree, that 0.5 times the standard hoagland nutrient solution in treatment N0.5 provides the plants with a relatively appropriate amount of nitrogen indeed. Analogously, there are several evidences that increasing nitrogen supply has led to increasing plant biomass in a nitrogen-deficient soil environment. For example, the biomass of sugarcane leaves can have been significantly increased along with increased nitrogen fertilizer(Jian, et al. 2011). Compared with the low nitrogen condition, paper birch in all groups under the high nitrogen condition has grown faster, their total biomass was larger, and their root/shoot ratio was lower. However, relatively larger root length has been found in no nitrogen treatment compared to other groups(Fig. 2B). Existing research suggests that plants invest more carbon assimilating substances into the root system to promote root growth and development, so as to obtain more restricted resources, resulting in an increase in root/shoot ratio of plants, when soil nutrients are scarce(Jian, et al. 2011). About the chlorophyll of perennial ryegrass in different concentration of nitrogen, the content under the condition of 0.5 times the standard hoagland nutrient solution is highest with a tendency to go up and then down in four treatments(Fig. 3). Previous research has shown that, under the stress of nitrogen deficiency, chlorophyll content of plant leaves decreases and F686 / F740 fluorescence ratio increases(Tartachnyk, et al. 2006). Excessive nitrogen transfer of nutrients in maize have resulted in premature leaf senescence and decreased photosynthetic capacity(Osaki, et al. 1995). Above all, it can be indicated that four kinds of nitrogen concentration designed to represent the conditions of no nitrogen, moderate amounts of nitrogen, a certain amount of excessive nitrogen and high concentration excessive nitrogen in different treatments is relatively reasonable. 


\section{DEGs related to nitrogen utilization and excessive nitrogen stress}

345 screened genes related to Nitrogen utilization generally display analogical expression patterns, which there was a rapidly increased expression treated with high concentration excessive nitrogen(Fig. 6A). A logical speculation that the expression of these genes will increase with the increase of nitrogen supply and their overexpression may cause a certain degree of disorder of nitrogen metabolism in plants under high concentration of excessive nitrogen stress, requires further verification. In addition, the expression patterns of 104 DEGs related to excessive nitrogen stress can be broadly divided into five categories(Fig. 6B). It means these genes may regulate plant response to excessive nitrogen stress in five corresponding ways.

\section{Special pathways that respond positively to different concentrations of nitrogen}

Photosynthesis-antenna proteins pathway come under observation under the condition of moderate amounts of nitrogen in KEGG enrichment analysis. 15 DEGs caused by moderate amounts of nitrogen application have been attributed to 2 categories, including $C A B 1 R$ (osa:4346803) and $C A B 2 R$ (osa:4324599), which could express two types of light-harvesting complex II chlorophyll a/b binding protein based on KEGG database. A previous study shows that OsCSP41b has an impact on leaf colors in rice(Mei, et al. 2017). It can be inferred that these genes are induced by moderate amounts of nitrogen and some analogously photosynthetic characteristics of perennial ryegrass display in this study. They may act as an important link between nitrogen and plant photosynthetic regulation mechanisms.

DEGs in carotenoid biosynthesis pathway has shown some enrichments under a certain amount of excessive nitrogen, including NCED/osa:4333566 and CCDlosa:4330451. OsNCED3 is involved in seed dormancy, plant growth, abiotic stress tolerance and leaf senescence by regulating the biosynthesis of $A B A$ in rice(Huang, et al. 2018). CitCCD4 makes $\beta$-cryptoxanthin and zeaxanthin into $\beta$-citraurin, leading to the flavedo of citrus fruit(Gang, et al. 2013). New functions of NCED and CCD selected in this study in excessive nitrogen stress remain to be explored.

The diverse parts of steroid biosynthesis pathway have been found to function in perennial ryegrass response to excessive nitrogen ranging from a certain concentration to high concentration, with CYP15G1|osa:4328054, SMT1|osa:4338803 to a certain concentration delta(7)-sterol-C5(6)desaturase/osa:4325687, probable 3-beta-hydroxysteroid-Delta(8) |osa:4326449, CYPlosa:4328054 to high concentration. Based on the previous studies, the application of ammonium sulfate advances to the high expression of FPPS, SMT1, SMT2, SMO1, SMO2, ODM and other pathway genes, accompanied by the increased content of sterol required for anilide biosynthesis in Withania somnifera (L.) Duna/(Pal, et al. 2017). CYP51s catalyzes the 14a-demethylation of sterol in all eukaryotes(Cabello-Hurtado, et al. 1997). We can deduce that these genes enriched in steroid biosynthesis pathway are indeed regulated by 
exogenous nitrogen, and then 14a-demethylation of sterol may be one of the key link between this pathway and excessive nitrogen stress.

\section{Transcription factors related to Nitrogen utilization and excessive nitrogen stress}

AP2-EREBP transcription factor family unique to plants is widely involved in the regulation of plant physiological functions and signal transduction pathways such as salicylic acid, jasmonic acid, abscisic acid and ethylene. AP2-EREBP transcription factors possess positive response to nitrogen, especially low nitrogen stress. This point has been demonstrated in many species and organs, including sugarcane(Yang, et al. 2019), leaves and roots of watermelon(Nawaz, et al. 2018), maize root(Xiujing, et al. 2016), Arabidopsis thaliana root(Ristova, et al. 2016) and maize(Chen, et al.). In this study, there was also a positive response to moderate amounts of nitrogen and excessive nitrogen stress though identifying these transcription factors. It advances a supplementary instruction that AP2-EREBP transcription factor family plays a functional regulatory role in plant response to different concentration of nitrogen. It is worth mentioning that ref0030344-processed-gene-0.2 from AP2-EREBP transcription factor family was regulated by both Nitrogen utilization and excessive nitrogen stress. The function of this TFs in regulating the molecular mechanisms of plant response to nitrogen deserves to be discussed in the future. MYB transcription factors are one of the largest families of transcription factors in plants, widely involved in the regulation of cell cycle(Mu, et al. 2009), secondary metabolism(Liu, et al. 2015), and biotic and abiotic stress(Nakabayashi, et al. 2014). SiMYB3 in Setaria italica plays a key role in regulating root growth, which in turn leads to increased tolerance to low-nitrogen stress(Ge, et al. 2019). Here, four MYB transcription factors have been identified to play some functional implication in Nitrogen utilization. This is consistent with our results.

NAC transcription factors not only participate in plant growth and development (such as lateral root development, flower development, secondary wall formation and plant hormone signal transduction, etc.), but also play an important regulatory role in plant response to abiotic stress such as drought, salinity, water logging and low temperature(Ye, et al. 2018). Some members in NAC transcription factor family perform the great importance in enhancing tolerance to N-deficiency in cotton(Magwanga, et al. 2019). In this study, one NAC transcription factor(ref0030817) has been found to exercise a regulatory function in excessive nitrogen stress. This may be a breakthrough to understand the role of the NAC transcription factor family in excessive nitrogen stress. Something similar happens in LOB and HB transcription factor families(novel101854,ref0036657). LOB transcription factors are mainly involved in the regulation of plant morphological development and photosynthesis. For example, MtLOB induced by nodule inception regulates the lateral root development in Medicago truncatula(Schiessl, et al. 2019). However, the potential regulatory mechanisms of LOB transcription factor on excessive nitrogen stress are unclear.

HB transcription factor is a very important regulatory protein in plants, which plays an important regulatory role in plant morphogenesis, hormone response, biological stress and abiotic stress response. $B v H b 1.2$ has high NOD-like activity in seeds, and $B v H b 1.1$ exhibits equal ability to combine NO in nitrate 
formation to protect chloroplasts from the deleterious effects of NO(Leiva, et al. 2019). In this study, HB transcription factor(ref0036657) screened to be related to excessive nitrogen stress, may be involved in some similar metabolic mechanisms of nitrite in perennial ryegrass, which could be a key point to guide and regulate nitrate accumulation in ryegrass.

\section{Conclusions}

At different concentration of nitrogen, a total of 101377 unigenes transcriptional characteristics were collected in perennial ryegrass by RNA-Seq, among which 345 and 104 DEGs were identified to be related to Nitrogen utilization and excessive nitrogen stress, separately.

KEGG enrichment analysis identified four significantly enriched pathways response to the nitrogen with different concentration, consisting of zeatin biosynthesis, photosynthesis-antenna proteins and isoquinoline alkaloid biosynthesis. In addition, 185 transcription factors from 37 transcription factor families, including the top three TF families(AP2-EREBP, MYB, NAC), were identified as TFs related to nitrogen. 22 transcription factors from 10 transcription factor families, especially AP2-EREBP and MYB transcription factor families were considered to perform some important regulatory functions in Nitrogen utilization. There were 4 transcription factors related to excessive nitrogen stress, coming from LOB, NAC, AP2-EREBP and HB transcription factor families. These results provided a new insight into the regulatory mechanism of the plants response to nitrogen utilization and excessive nitrogen stress.

\section{Methods}

\section{Plant materials}

The plant material used in this research was perennial ryegrass "Taya" (variety registration no.:285), which was introduced by professor Liebao Han of Beijing forestry university from DLF SEEDS A/S company in Denmark on December 8, 2004. The seeds of perennial ryegrass were preserved at the Lawn Research Institute of Beijing Forestry University(Beijing, China). After a week of vernalization in a $4^{\circ} \mathrm{C}$ refrigerator, the seeds were placed on petri dishes with sterile filter paper for germination under natural conditions in the laboratory. After two weeks of seed cultivation, seedlings about $5 \mathrm{~cm}$ in height were selected and suspended in hoagland nutrient solution with nitrogen control(tableS3). In a chamber with $16 \mathrm{~h}$ light/ $8 \mathrm{~h}$ darkness at $23^{\circ} \mathrm{C}$, the seedlings were grown in hoagland nutrient solution with $0,0.5,1$, and 10 times the standard concentration of nitrogen for 4 weeks(table S4). The treatments were called $\mathrm{N} 0, \mathrm{~N} 0.5, \mathrm{~N} 1, \mathrm{~N} 10$ in turn. Three plants of each treatment were randomly selected as biological replicates. A total of 12 samples were immediately treated with liquid nitrogen and stored at $-80^{\circ} \mathrm{C}$.

\section{The determination of phenotypic characterization}

Four plants were randomly selected from each treatment and their plant height, root length, fresh weight and tiller number were measured respectively. About $0.1 \mathrm{~g}$ of each sample was placed in $95 \%$ alcohol, then stood in the dark for $24 \mathrm{~h}$ and the specific mass was recorded. The absorbance values of these 
samples were measured at wavelengths of 665,649 and $470 \mathrm{~nm}$ and were corrected with $95 \%$ alcohol. The contents of chlorophyll $\mathrm{a} / \mathrm{b}$ and total chlorophyll were calculated by corresponding formulas(Wellburn and R. 1994).

\section{RNA isolation and detection}

Total plant RNA was extracted using the oxygen RNA extraction kit and then treated with the RNase-Free DNasel Set to remove contaminating genomic DNA. Four methods were used to detect the quality of RNA as follows. Agarose gel electrophoresis was used to analyze the degradation degree and contamination of RNA. The purity of RNA was detected by Nanodrop (od260/280 ratio). RNA concentration was accurately quantified by Qubit. The integrity of RNA was detected by Agilent 2100 precisely.

\section{Library construction for transcriptome sequencing}

After the sample passed the test, mRNA of samples was enriched by magnetic beads with Oligo (dT) binding with polyA tail of mRNA through a-t complementary pairing. Secondly, mRNA was fragmented into short segments using fragmentation buffer. Using mRNA as template, a strand of cDNA was synthesized using random hexamers, and then two strand cDNA was synthesized using buffer, dNTPs and DNA polymerase I. AMPure XP beads were used to purify double strand CDNA. Purified doublestranded cDNA was repaired at the end, followed by adding A tail and connecting sequencing joints. AMPure XP beads were used to select each segment size. Finally, PCR enrichment was carried out to obtain the final cDNA library.

After the construction of the library, Qubit2.0 was used for initial quantification, and the library was diluted to $1 \mathrm{ng} / \mathrm{ul}$, and then the insert size of the library was detected by Agilent 2100. After the insert size met the expectation, qPCR method was used to accurately quantify the effective concentration of the library $(>2 \mathrm{nM})$ to ensure the quality of the library.

HiSeq sequencing was carried out after pooling different libraries in accordance with the requirements of effective concentration and target disembarkation data.

\section{Quality control, reads mapping and functional annotation}

The original image data files obtained by high-throughput sequencing (such as Illumina HiSeqTM) were transformed into Sequenced Reads, called raw data by CASAVA Base Calling analysis. The error rate of each base sequencing was converted by sequencing Phred value (Phred score, Qphred) through the formula (formula 1: Qphred $=-10 \log 10(e)$ ). Segregating of base A/T and C/G or not was confirmed by base $\mathrm{C} / \mathrm{G}$ content distribution test. To Ensure the quality of information analysis, we filtered out Low quality reads with connectors from raw data and got the new data called clean reads for further analysis.

HISAT software was selected to localize the filtered sequences. Clean reads were compared to the reference genome. Reads distribution in different regions of the reference genome was counted, including those that align to exon occupied the highest content and intron derived from pre-mRNA residues and intron retention during the alternative splicing. The density of Total mapped reads mapped to each 
chromosome in the genome (plus or minus chains) was analyzed. Integrative Genomics Viewer was used to visually browse the bam files containing RNA-seq reads for genomic alignment and corresponding reference genome and annotation files.

\section{Analysis of differentially expressed genes(DEGs)}

It adopted DESeq(Anders, et al. 2015) to normalize read count first and then the hypothesis testing probability ( $p$-value) was calculated according to the model. Finally, multiple hypothesis testing and correction were performed to obtain the value of FDR (error detection rate). The screening criteria for differentially expressed genes were pval $<0.05$ and $\mid \log 2$ (FoldChange) $\mid>1$. The overall distribution of differential genes were inferred from the volcano map. The number of differentially expressed genes and the overlap between the comparison groups were shown by using the Venn diagram. The FPKM value of the differentially expressed genes under different experimental conditions was taken as the expression level for hierarchical clustering analysis. The relative expression level log2(ratios) of differentially expressed genes were clustered by h-cluster, k-means and SOM. Different clustering algorithms divide the differential genes into several clusters, and the genes in the same cluster have similar expression level variation trend under different processing conditions.

\section{GO and KEGG enrichment analysis of differentially expressed genes}

GOseq(Young, et al. 2010) was the software method used in the GO enrichment analysis, which was based on Wallenius non-central geometric distribution. GO terms with corrected Pvalue less than 0.05 were considered significantly enriched by differential expressed genes.

On the other hand, Pathway in KEGG database was taken as the unit, and hypergeometric test was applied to find out the Pathway significantly enriched in differentially expressed genes compared with the whole genomic background. We used KOBAS software to test the statistical enrichment of differential expression genes in KEGG pathways.

\section{Transcription factors analysis}

ITAK software was used to predict transcription factors in differentially expressed genes.

TF(transcription factor) family and rules defined in database were used to identify TF by hmmscan(Paulino, et al. 2009).

\section{Quantitative Real-Time PCR analysis}

24 unigenes were selected for quantitative Real-Time PCR analysis so as to verify the accuracy of transcriptome data and the actual expression of related genes. The previously extracted total RNA was reversely transcribed into cDNA as a qPCR template. All primers designed with Primer Premier 5.0 software are displayed. Ryegrass actin was used as an internal reference gene to calculate the relative expression level. Quantitative Real-Time PCR analysis was performed by bio-rad CFX connected server 
system. Reaction conditions were set as follows: $95^{\circ} \mathrm{C}$ for $10 \mathrm{~min} ; 95^{\circ} \mathrm{C} 15 \mathrm{~s}, 60^{\circ} \mathrm{C} 1 \mathrm{~min}, 40 \mathrm{cycles}$. Each reaction was completed with four technical replicates. The relative expression levels of all genes were determined by the 2-deltadelta Ct method(Livak and Schmittgen 2000).

\section{List Of Abbreviations}

TFs: transcription factors

KEGG: Kyoto Encyclopedia of Genes and Genomes

GO: Gene Ontology

DEGs: differentially expressed genes

qRT-PCR: Quantitative Real-Time PCR

\section{Declarations}

Ethics approval and consent to participate

Not applicable

\section{Consent for publication}

Not applicable

\section{Availability of data and materials}

The datasets generated and/or analyzed during the current study will be available in the NCBI repository after 1 years.

The datasets used and/or analyzed during the current study are available from the corresponding author on reasonable request.

\section{Competing interests}

The authors declare that they have no competing interests.

\section{Funding}

The program was supported by the National Natural Science Foundation of China (No. 31672477) and Beijing Natural Science Foundation (No.6204039). Both of them provide funding for the entire research effort, which is used for material maintenance, reagents, data collection, labor costs and so on.

\section{Author contribution}


Yinruizhi Li and Yuehui Chao proposed ideas and designed the experiments. Yinruizhi Li and Mengdi Wang completed the experiments. Yinruizhi Li and Ke Teng analyzed the experimental data. Di Dong, Zhuocheng Liu and Tiejun Zhang put forward key opinions that cannot be ignored. Yinruizhi Li and Yuehui Chao wrote the manuscript. Liebao Han played a guiding role in the whole work process. All authors read and reached an agreement with the final manuscript.

\section{Acknowledgements}

Not applicable

\section{References}

Anders S, Pyl PT, Huber W. HTSeq-a Python framework to work with high-throughput sequencing data. BIOINFORMATICS 31:166-169

Bijay-Singh, Yadvinder-Singh, Sekhon GS. Fertilizer-N use efficiency and nitrate pollution of groundwater in developing countries. j contaminant hydrology 20:167-184

Bijay-Singh, Yadvinder-Singh, Sekhon GS. Fertilizer-N use efficiency and nitrate pollution of groundwater in developing countries. ENVIRON POLLUT 3:366

Byrne SL, Nagy I, Pfeifer M, Armstead I, Asp T. A synteny-based draft genome sequence of the forage grass Lolium perenne. PLANT J 84:816-826

Cabello-Hurtado F, Zimmerlin A, Rahier A, Taton M, DeRose R, Nedelkina S, Batard Y, Durst F, Pallett KE, Werck-Reichhart. Cloning and Functional Expression in Yeast of a cDNA Coding for an Obtusifoliol 14aDemethylase (CYP51) in Wheat. BIOCHEM BIOPH RES CO 2:381-385

Chen Q, Liu Z, Wang B, Wang X, Lai J, Tian F Transcriptome sequencing reveals the roles of transcription factors in modulating genotype by nitrogen interaction in maize. PLANT CELL REP 34:1761-1771

Gang M, Lancui Z, Asami M, Kazuki M, Kazuki Y, Masaki Y, Anung W, Reiko M, Kato M. Enzymatic Formation of $\beta$-Citraurin from $\beta$-Cryptoxanthin and Zeaxanthin by Carotenoid Cleavage Dioxygenase4 in the Flavedo of Citrus Fruit. PLANT PHYSIOL 2:682-695

Ge L, Dou Y, Li M, Qu P, He Z, Liu Y, Ma Y. SiMYB3 in Foxtail Millet (Setaria italica) Confers Tolerance to Low-Nitrogen Stress by Regulating Root Growth in Transgenic Plants. International Journal of Molecular Sciences 22:5741

Gelli M, Konda AR, Liu K, Zhang C, Dweikat IM. Validation of QTL mapping and transcriptome profiling for identification of candidate genes associated with nitrogen stress tolerance in sorghum. BMC PLANT BIOL $17: 123$ 
Hu R, Yu C, Wang X, Jia C, Pei S, He K, He G, Kong Y, Zhou G. De novo Transcriptome Analysis of Miscanthus lutarioriparius Identifies Candidate Genes in Rhizome Development. FRONT PLANT SCI 8:492

Huang A, Sang Y, Sun W, Fu Y, Yang Z. Transcriptomic Analysis of Responses to Imbalanced Carbon: Nitrogen Availabilities in Rice Seedlings. PLOS ONE 11:e165732

Huang Y, Guo Y, Liu Y, Zhang F, Wang Z, Wang H, Wang F, Li D, Mao D, Luan S, Liang M, Chen L. 9-cisEpoxycarotenoid Dioxygenase 3 Regulates Plant Growth and Enhances Multi-Abiotic Stress Tolerance in Rice. FRONT PLANT SCI 9:162

Jian RW, Hawkins CD, Letchford T. Relative growth rate and biomass allocation of paper birch (Betula papyrifera) populations under different soil moisture and nutrient regimes. CAN J FOREST RES 28:44-55

Kotur Z, GLASS ADM. A $150 \mathrm{kDa}$ plasma membrane complex of AtNRT2.5 and AtNAR2.1 is the major contributor to constitutive high-affinity nitrate influx in Arabidopsis thaliana. Plant Cell \& Environment 38:1490-1502

Leiva EN, Reeder BJ, Wilson MT, Bulow L. Sugar beet hemoglobins: reactions with nitric oxide and nitrite reveal differential roles for nitrogen metabolism. BIOCHEM J 476:2111-2125

Liu Y, Shi Z, Maximova SN, Payne MJ, Guiltinan MJ. Tc-MYBPA an Arabidopsis TT2-like transcription factor and functions in the regulation of proanthocyanidin synthesis in Theobroma cacao. BMC PLANT BIOL 15:160

Livak K, Schmittgen T. Analysis of Relative Gene Expression Data Using Real-Time Quantitative PCR and the 2- $\triangle \triangle$ Ct Method. METHODS 25:402-408

Loaiza P, Balocchi O, de la Barra C, López I F. Perennial ryegrass productivity and nutritive quality as affected by frequency of nitrogen fertilizer addition. GRASSL SCI 2:86-92

Lu M, Chen M, Song J, Wang Y, Pan Y, Wang C, Pang J, Fan J, Zhang Y. Anatomy and transcriptome analysis in leaves revealed how nitrogen $(\mathrm{N})$ availability influence drought acclimation of Populus. Trees

Magwanga RO, Kirungu JN, Lu P, Cai X, Zhou Z, Xu Y, Liu F. Map-Based Functional Analysis of the GhNLP Genes Reveals Their Roles in Enhancing Tolerance to N-Deficiency in Cotton. INT J MOL SCI 19:4953

McClearn B, Gilliland TJ, Delaby L, Guy C, Dineen M, Coughlan F, McCarthy B. Milk production per cow and per hectare of spring-calving dairy cows grazing swards differing in Lolium perenne L. ploidy and Trifolium repens L. composition. J DAIRY SCI 102:8571-8585

Mei J, Li F, Liu X, Hu G, Fu Y, Liu W. Newly identified CSP41b gene localized in chloroplasts affects leaf color in rice. PLANT SCI 256:39-45 
Mu RL, Cao YR, Liu YF, Lei G, Zou HF, Liao Y, Wang HW, Zhang WK, Ma B, Du JZ, Yuan M, Zhang JS, Chen SY. An R2R3-type transcription factor gene AtMYB59 regulates root growth and cell cycle progression in Arabidopsis. CELL RES 19:1291-1304

Nakabayashi R, Yonekura-Sakakibara K, Urano K, Suzuki M, Yamada Y, Nishizawa T, Matsuda F, Kojima M, Sakakibara H, Shinozaki K, Michael AJ, Tohge T, Yamazaki M, Saito K. Enhancement of oxidative and drought tolerance in Arabidopsis by overaccumulation of antioxidant flavonoids. PLANT J 77:367-379

Nawaz MA, Chen C, Shireen F, Zheng Z, Sohail H, Afzal M, Ali MA, Bie Z, Huang Y. Genome-wide expression profiling of leaves and roots of watermelon in response to low nitrogen. BMC GENOMICS 19:456

Osaki M, lyoda M, Tadano T. Ontogenetic changes in the contents of ribulose-1,5-bisphosphate carboxylase/oxygenase, phosphoenolpyruvate carboxylase, and chlorophyll in individual leaves of maize. Soil Science \& Plant Nutrition 41:285-293

Pal S, Yadav AK, Singh AK, Rastogi S, Gupta MM, Verma RK, Nagegowda DA, Pal A, Shasany AK. Nitrogen treatment enhances sterols and withaferin A through transcriptional activation of jasmonate pathway, WRKY transcription factors, and biosynthesis genes in Withania somnifera (L.) Dunal. PROTOPLASMA 254:389-399

Paulino PR, Mauricio RPD, Guedes CLG, Rensing SA, Birgit K, Bernd MR. PInTFDB: updated content and new features of the plant transcription factor database. NUCLEIC ACIDS RES 1 38(suppl_1)囚 D822-D827

Peng M, Bi YM, Zhu T, Rothstein S. Genome-wide analysis of Arabidopsis responsive transcriptome to nitrogen limitation and its regulation by the ubiquitin ligase gene NLA. PLANT MOL BIOL 65:775-797

Ristova D, Carre C, Pervent M, Medici A, Kim GJ, Scalia D, Krouk G. Combinatorial interaction network of transcriptomic and phenotypic responses to nitrogen and hormones in the Arabidopsis thaliana root. SCI SIGNAL 451:s13

Ruuska SA, Lewis DC, Kennedy G, Furbank RT, Jenkins CL, Tabe LM. Large scale transcriptome analysis of the effects of nitrogen nutrition on accumulation of stem carbohydrate reserves in reproductive stage wheat. PLANT MOL BIOL 66:15-32

SchiessI K, Lilley JLS, Lee T, Tamvakis I, Kohlen W, Bailey PC, Oldroyd GED. NODULE INCEPTION Recruits the Lateral Root Developmental Program for Symbiotic Nodule Organogenesis in Medicago truncatula. CURR BIOL

Tartachnyk II, Rademacher I, Kühbauch W. Distinguishing nitrogen deficiency and fungal infection of winter wheat by laser-induced fluorescence. PRECIS AGRIC 7:281-293

Tiwari JK, Buckseth T, Zinta R, Saraswati A, Singh RK, Rawat S, Dua VK, Chakrabarti SK. Transcriptome analysis of potato shoots, roots and stolons under nitrogen stress. Sci Rep 10:1152 
Wang L, Jiang J, Song A, Wang H, Li P, Guan Z, Chen F, Chen S. Comparative transcriptome analysis of Chrysanthemum nankingense in response to nitrogen deficiency. SCI HORTIC-AMSTERDAM 195:101-107

Wang YY, Cheng YH, Chen KE, Tsay YF. Nitrate Transport, Signaling, and Use Efficiency. ANNU REV PLANT BIOL 69:85-122

Wang YY, Hsu P, Tsay Y Uptake, allocation and signaling of nitrate. TRENDS PLANT SCI 17:458-467

Wellburn, R. A. The Spectral Determination of Chlorophylls a and b, as well as Total Carotenoids, Using Various Solvents with Spectrophotometers of Different Resolution. J PLANT PHYSIOL 144:307-313

Wright, J. M. Nitrate Accumulation In Crops And Nitrate Poisoning In Animals. Advances in Agronomy 197-247

Xiujing H, Haixia M, Xiongwei Z, Shujun N, Yuhua L, Zhiming Z, Yaou S, Qi C, Yanli L, Hai L, Shufeng Z, Shibin G, Guangtang P, Haijian L. Comparative RNA-Seq Analysis Reveals That Regulatory Network of Maize Root Development Controls the Expression of Genes in Response to N Stress. PLOS ONE $11: \mathrm{e} 151697$

Yang Y, Feng W, Qing W, Jianyun R. Transcriptome analysis using RNA-Seq revealed the effects of nitrogen form on major secondary metabolite biosynthesis in tea (Camellia sinensis) plants. ACTA PHYSIOL PLANT 7:127

Yang Y, Gao S, Su Y, Lin Z, Guo J, Li M, Wang Z, Que Y, Xu L. Transcripts and low nitrogen tolerance: Regulatory and metabolic pathways in sugarcane under low nitrogen stress. ENVIRON EXP BOT

Ye G, Ma Y, Feng Z, Zhang X. Transcriptomic analysis of drought stress responses of sea buckthorn (Hippophae rhamnoidessubsp. sinensis) by RNA-Seq. PLOS ONE 8:e202213

Young MD, Wakefield MJ, Smyth GK, Oshlack A. Gene ontology analysis for RNA-seq: accounting for selection bias. GENOME BIOL 11:R14

\section{Supplementary Table Legends}

\section{Table S1}

The quality of data output

\section{Table S2}

Information of reads mapped to genome

\section{Table S3}

The components of standard hoagland nutrient solution. 
Table S4

The nutrient element components in different treatments.

\section{Figures}

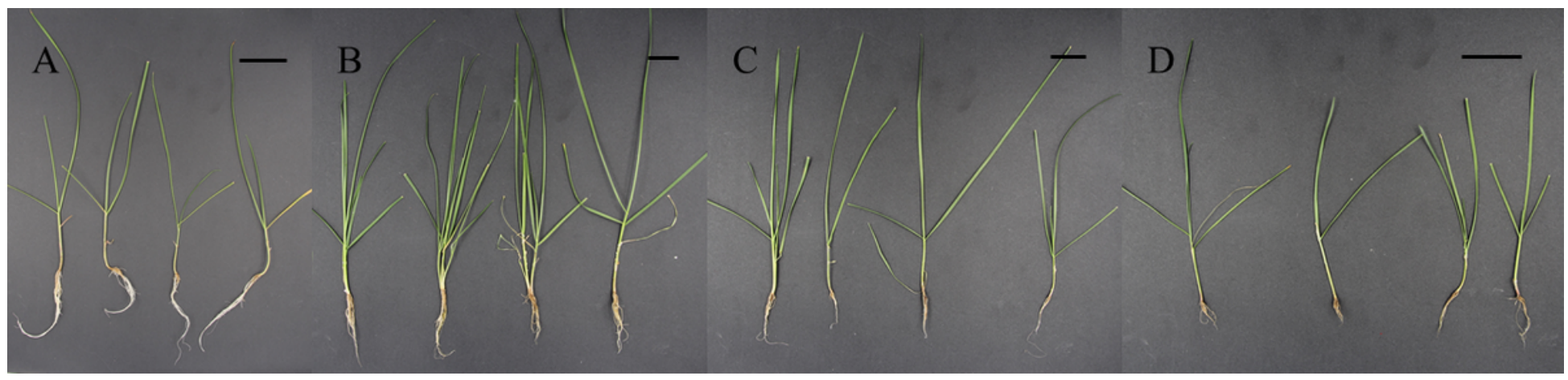

\section{Figure 1}

The growth state of perennial ryegrass under different treatments. A-D represents treatment N0, N0.5, N1, N10 in turn. Black scale in each figure means $3 \mathrm{~cm}$.

A

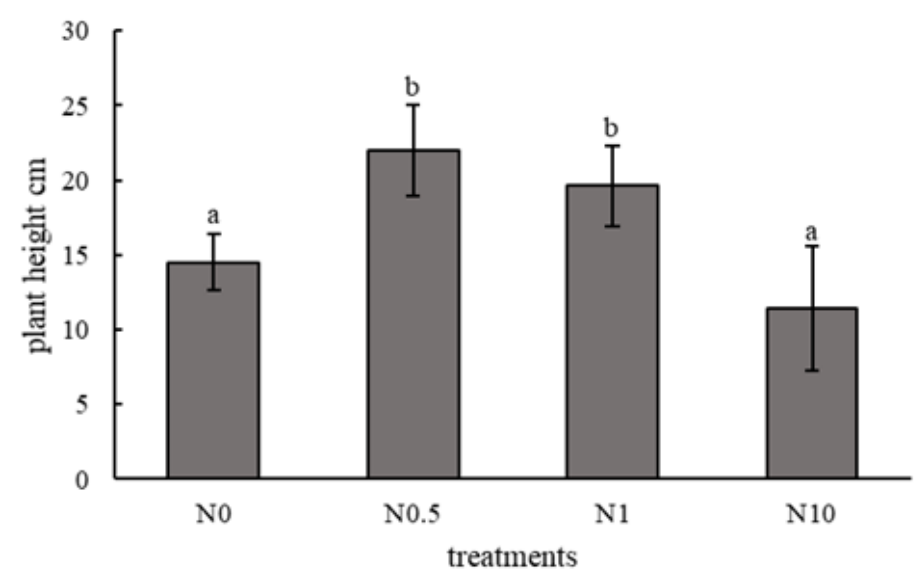

$\mathrm{C}$

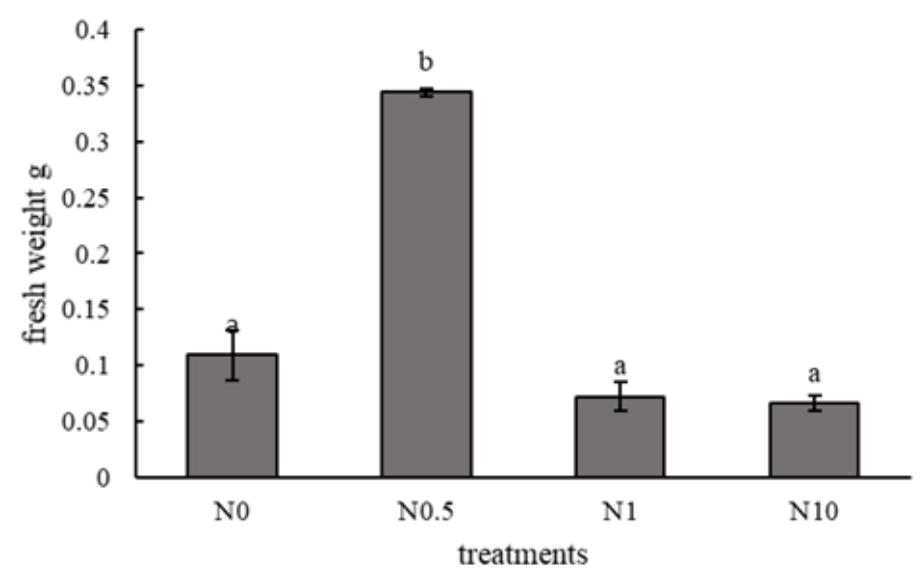

B

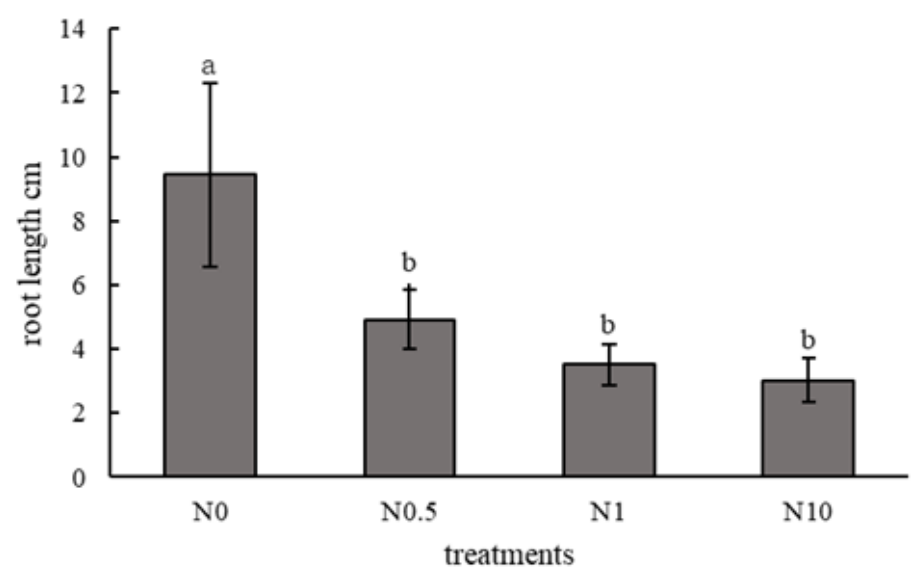

$\mathrm{D}$

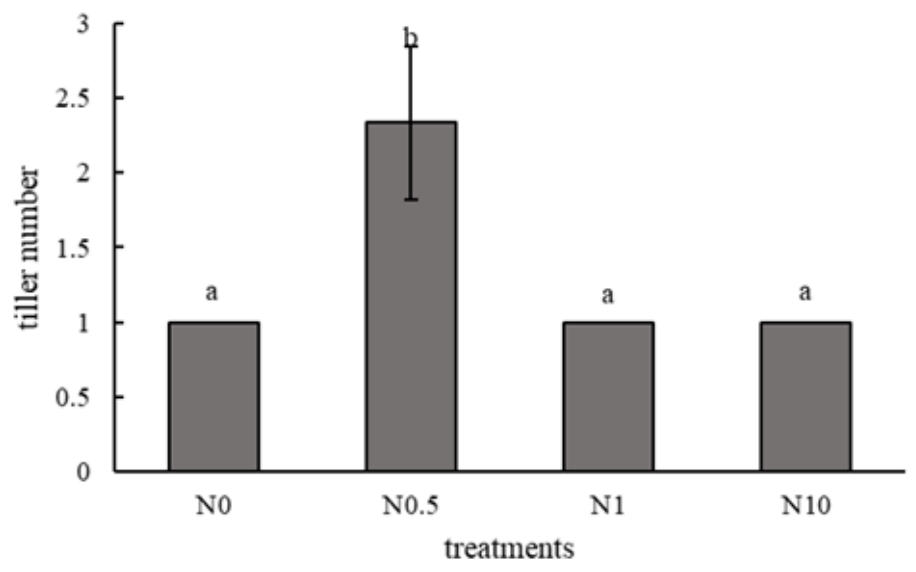


Figure 2

A-D represents plant height, root length, fresh weight and tiller number of different treatments in turn.

$\mathrm{E}$

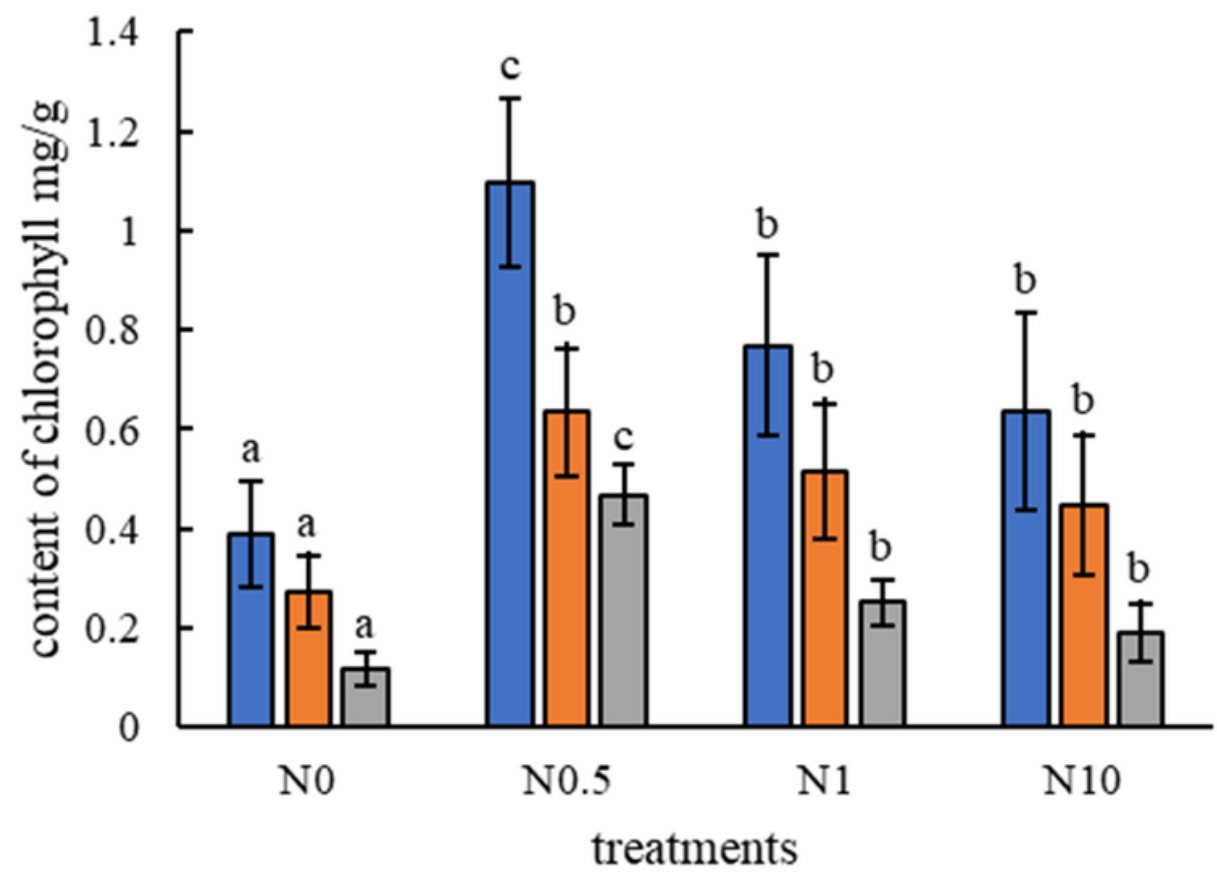

$\square$ The total chlorophyll

口chlorophyll a

口chlorophyll b

\section{Figure 3}

The content of chlorophyll. The blue pillars means total chlorophyll content. The yellow and grey pillars mean the content of chlorophyll $a$ and $b$ separately. $a$ and $b$ in all figures represents significant differences and error bars mean differences between biological replications. 
A

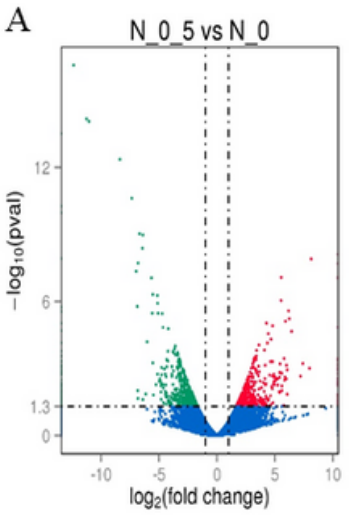

D

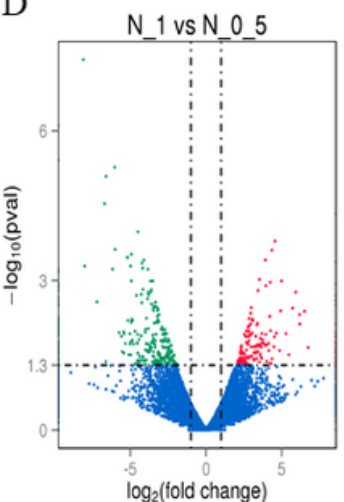

Differential Expressed Genes ( 883 )

- up regulated: 422

- down regulated: 461

Differential Expressed Genes ( 456

- up regulated: 169

down regulated: 287
B

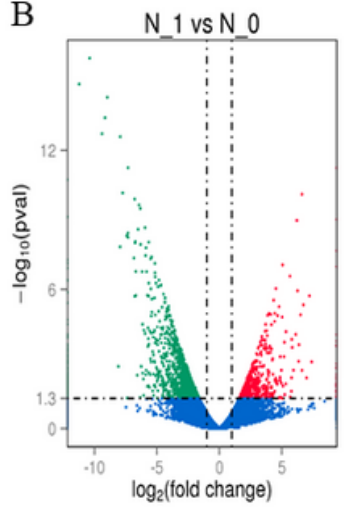

E

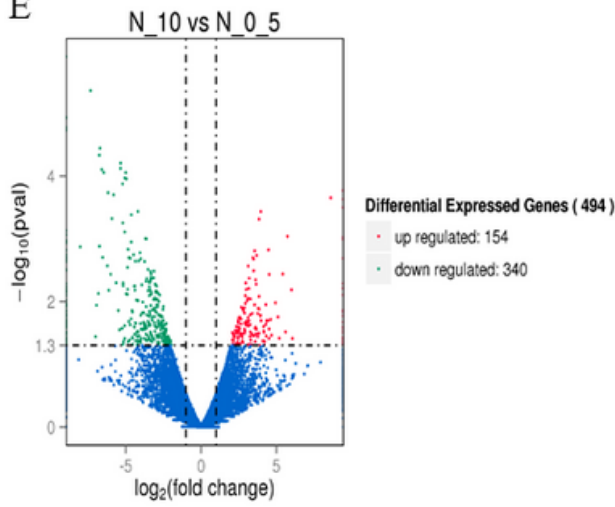

C

(1597)

up regulated: 546

down regulated: 1051

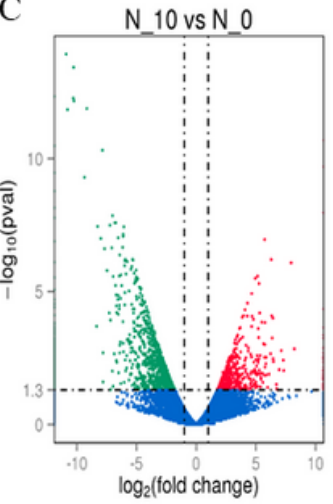

Differential Expressed Genes (1778)

up regulated: 612

down regulated: 1166

\section{Figure 4}

DEGs volcano map. A-C represents DEGs in three trentments(N0.5, N1 and N10) with treatment N0 as control. D-E represents DEGs in two trentments(N1 and N10) with treatment N0.5 as control. Genes with significant differentially expression are represented by red dots (up-regulated) and green dots (downregulated), while genes without significant differentially expression are represented by blue dots. The abscissa represents the multiple change of gene expression in different samples. The ordinate represents the statistical significance of the difference in gene expression.

a

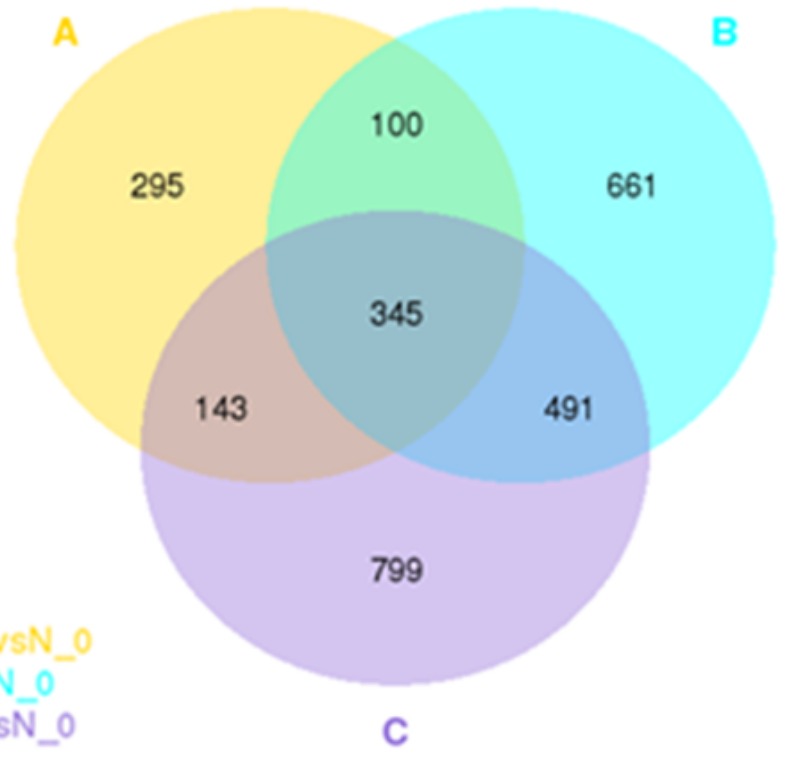

b
N_10vsN_0_5 N_1vsN_0_5

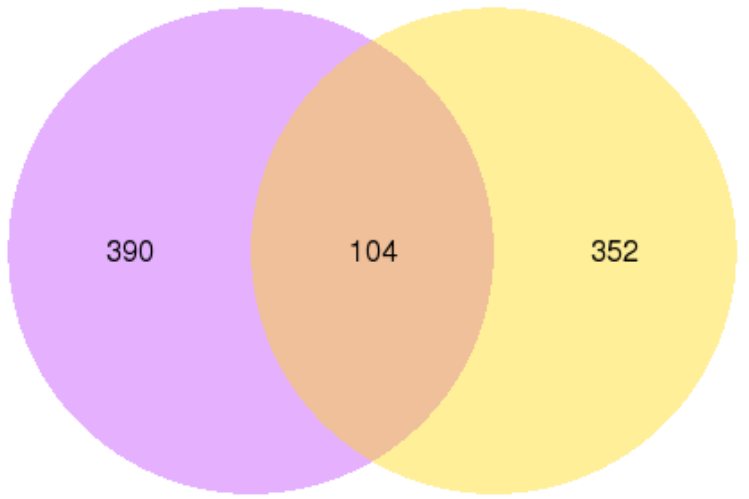




\section{Figure 5}

Venn diagram of DEGs. a represents DEGs in three other groups compared to treatment $\mathrm{N} 0$ as control. b represents DEGs in trentments $\mathrm{N} 1$ and N10 compared to treatment N0.5 as control. The sum of the Numbers in each large circle represents the total number of differentially expressed genes in the comparison combination, and the overlapping circles represent the number of differentially expressed genes in common between the combinations.

A Unigenes related to nitrogen absorption

$-1.0$

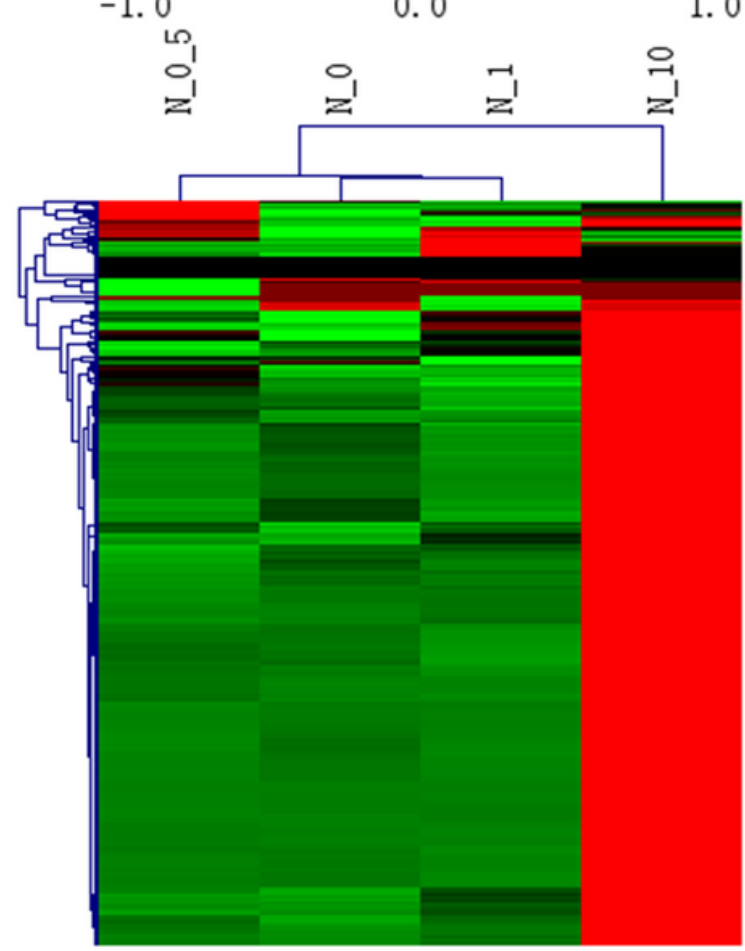

B Unigenes related to excessive nitrogen stress
0.0

1. 0

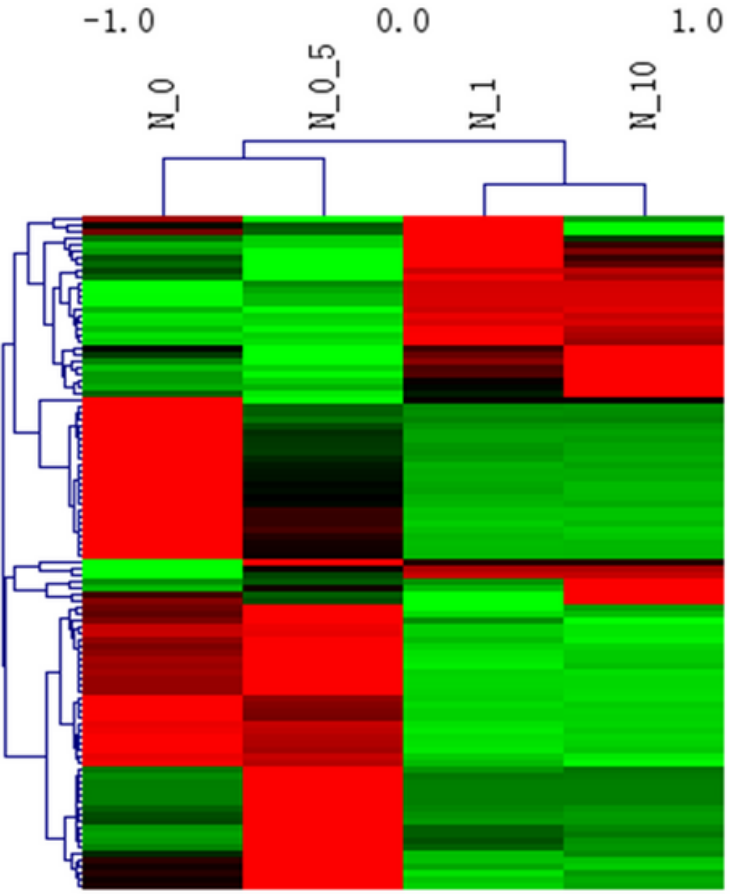

\section{Figure 6}

A and $B$ represents the clustering situation of unigenes related to Nitrogen utilization and excessive nitrogen stress, respectively. High expression genes are shown in red and low expression genes are shown in blue. 

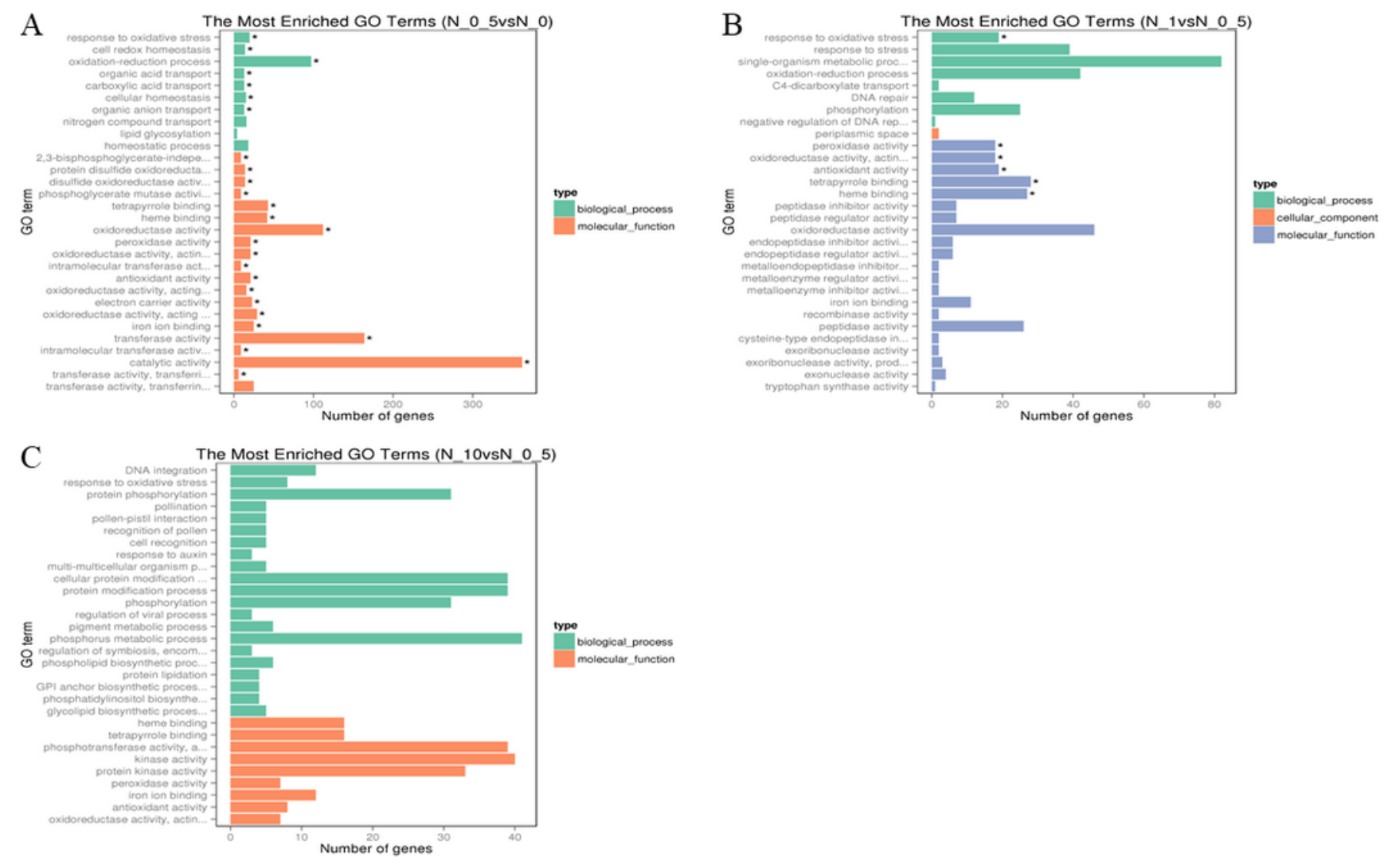

\section{Figure 7}

GO enrichment analysis of DEGs. A represents GO enrichment of DEGs in the treatment N0.5 compared to treatment N0 as control. B-C represents GO enrichment of DEGs in the treatment N1 and N10 compared to treatment N0.5 as control. The ordinate is the enriched GO term, and the abscissa is the number of differentially expressed genes in the term. Different colors were used to distinguish biological processes, cell components and molecular functions, and the * was a significantly enriched GO term. 

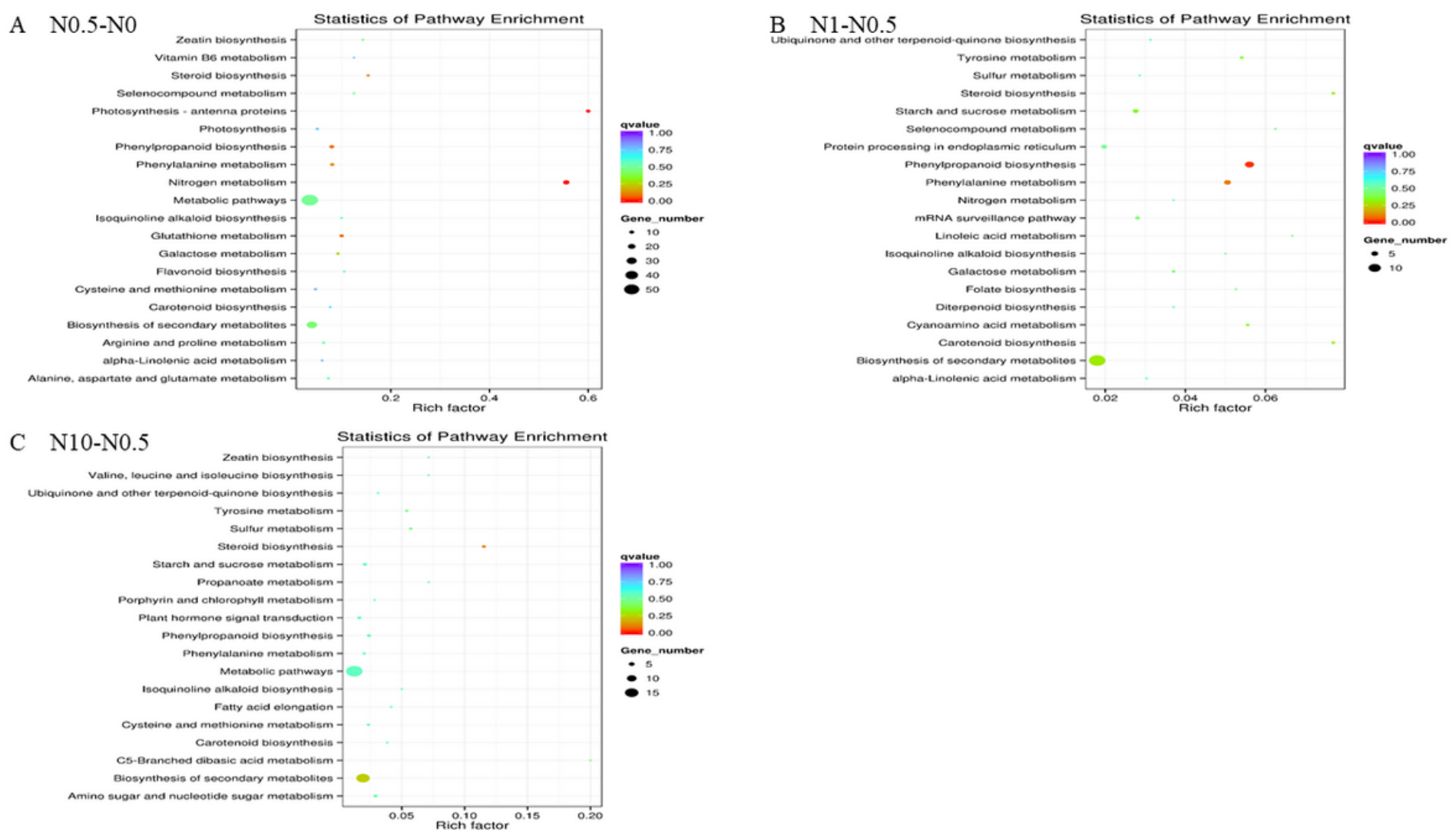

\section{Figure 8}

KEGG enrichment distribution of DEGs. The vertical axis represents pathway name, the horizontal axis represents Rich factor, the size of the dots represents the number of differentially expressed genes in the pathway, and the color of the dots corresponds to different Qvalue ranges.

A Photosynthesis-antenna proteins(N0.5-N0)

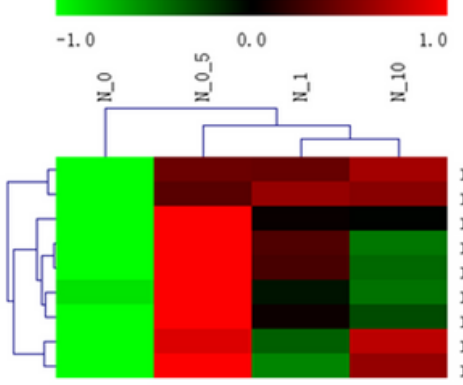

ref0019375-processed-gene-0. 1 |osa: 4346803 ref0019375-processed-gene-0. 4 osa: 4346803 ref0001049-processed-gene- 0.0 osa: 4346803 ref0036539-processed-gene-0. 3 osa: 4324599 ref0003114-snap-gene-0. 32 |osa: 4324599 ref0038731-processed-gene-0.4 4 osa: 4346803 ref0045877-processed-gene-0. 3 osa: 4324599 ref0045877-processed-gene-0.3 osa: 4324
ref0023193-snap-gene-1. 26 losa: 4346803 ref0023193-snap-gene-1. 26 losa: 4346803
ref0023193-processed-gene-1. 7 |osa: 4346803

C Steroid biosynthesis (N1-N0.5)

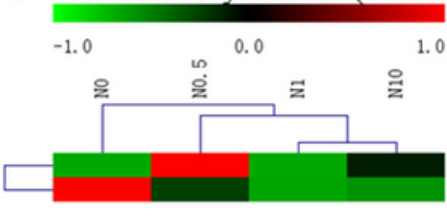

ref0042161-snap-gene-0. 33|osa: 4328054 ref0043399-snap-gene-0. 9 |osa: 4338803
B Carotenoid biosynthesis (N1-N0.5)

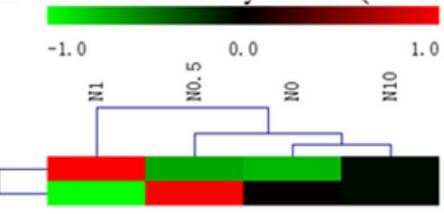

ref0000551-processed-gene- 0.4 losa: 4333566 ref0019293-processed-gene-0. 6 osa: 4330451

D Steroid biosynthesis (N10-N0.5)

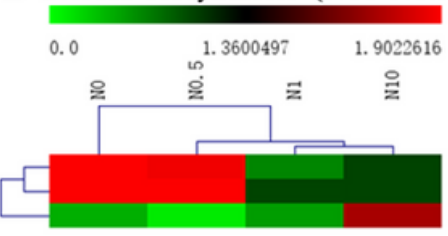

ref0046041-snap-gene-0. 4 losa: 4325687 ref0017330-snap-gene-0.5 ref0014948

\section{Figure 9}

A represents the clustering situation of DEGs, enriched in photosynthesis-antenna proteins pathway, in treatment N0.5 compared to treatment NO. B-C represents the clustering situation of DEGs, enriched in steroid biosynthesis pathway and carotenoid biosynthesis pathway, in treatment $\mathrm{N} 1$ compared to 
treatment N0.5. D represents the clustering situation of DEGs, enriched in steroid biosynthesis pathway, in treatment N10 compared to treatment N0.5. High expression genes are shown in red and low expression genes are shown in blue.

A

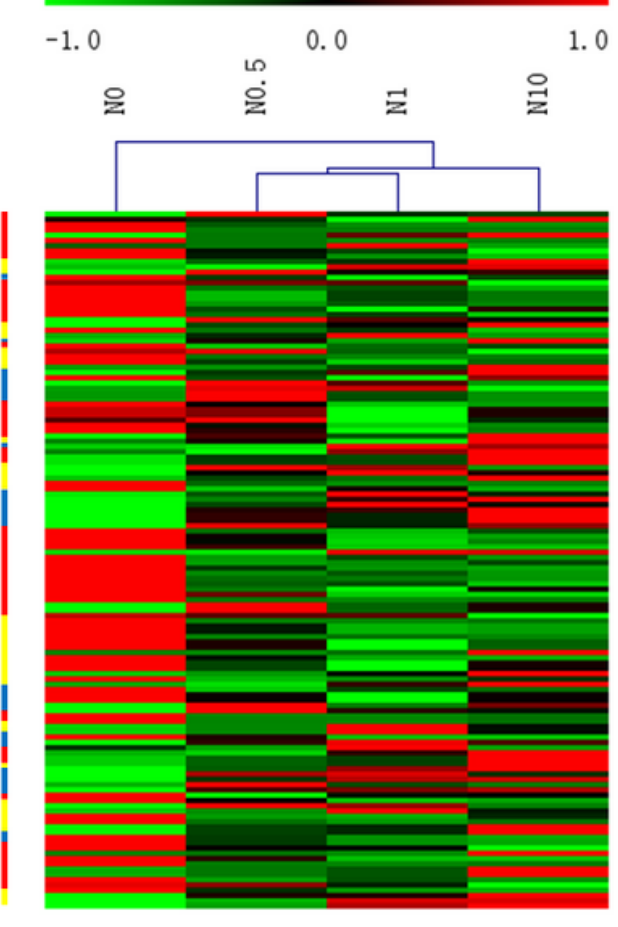

ABI3VP1

AP2-EREBP

ARF

AUX/LAA

bHLH

bZIP

C2 2 -CO-like

C2C2-Dof

$\mathrm{C} 2 \mathrm{H} 2$

$\mathrm{C} 3 \mathrm{H}$

CAMTA

CCAAT

FAR1

G2-like

GNAT

GRAS

HB

HSF

Jumonji

LFY

LOB

MADS

MTERF

MYB

NAC

Orphans

PBF-2-like

PLATZ

RWP-RK

SRS

SWI/SNF-BAF60b

TCP

Tify

TRAF

TUB

WRKY

zf-HD

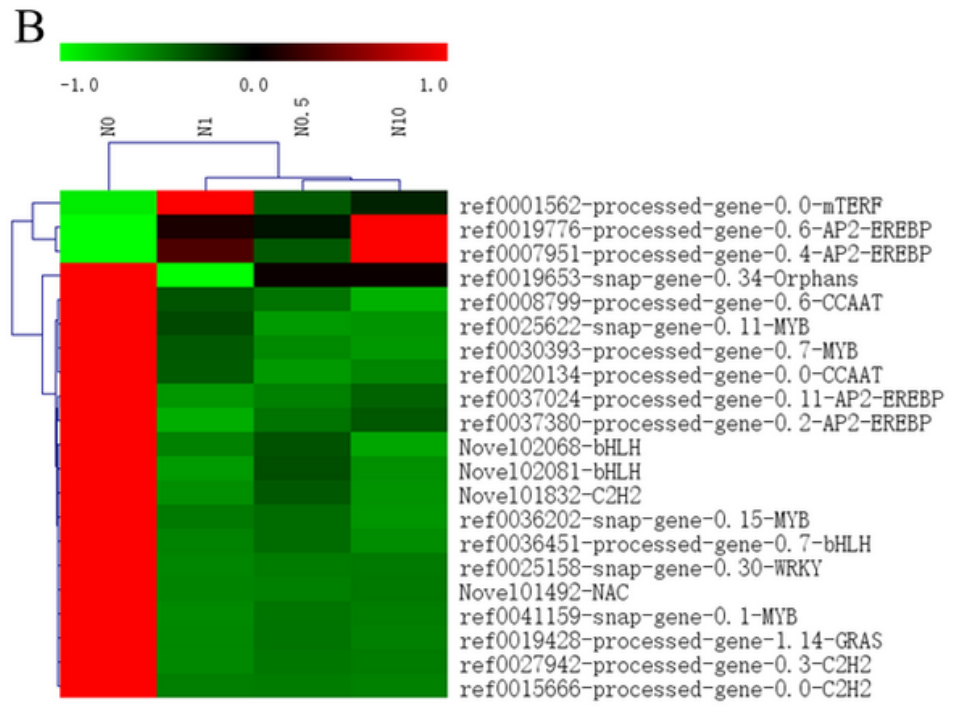

C

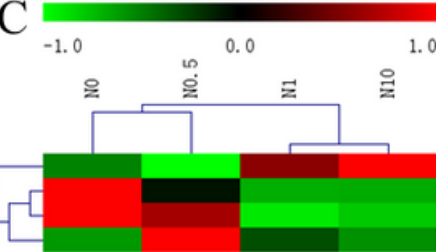

Nove101854-LOB

ref0030817-snap-gene-0. 18-NAC ref0030344-processed-gene-0. 2-AP2-EREBP ref0036657-snap-gene-0. 9-HB

\section{Figure 10}

A represents the clustering situation of TFs response to nitrogen in perennial ryegrass. B represents the clustering situation of TFs related to Nitrogen utilization. C represents the clustering situation of TFs related to excessive nitrogen stress. Color pillars mean the different transcription factor families in turn and the length of them means the number of TFs. High expression genes are shown in red and low expression genes are shown in blue. 
A

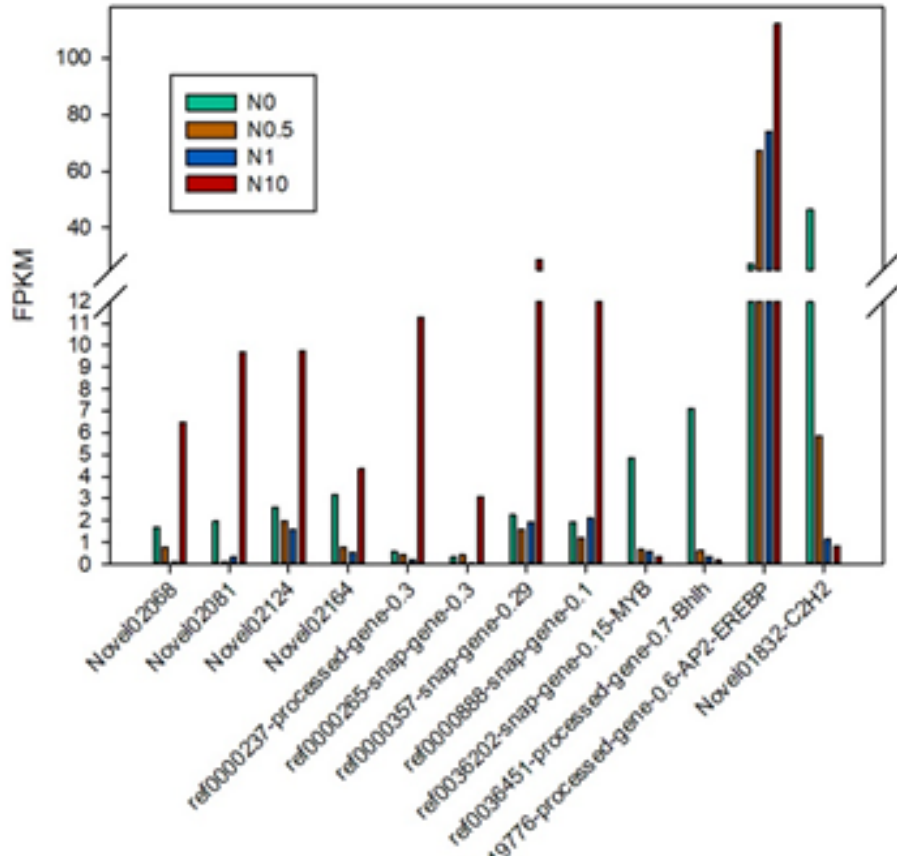

$\mathrm{C}$

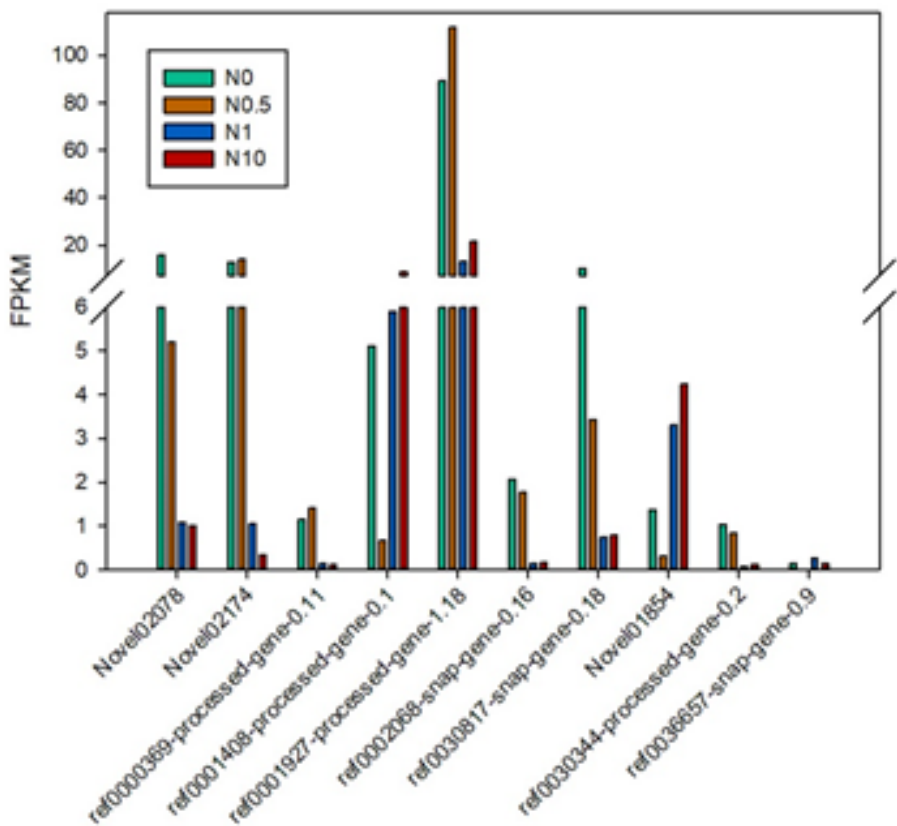

B

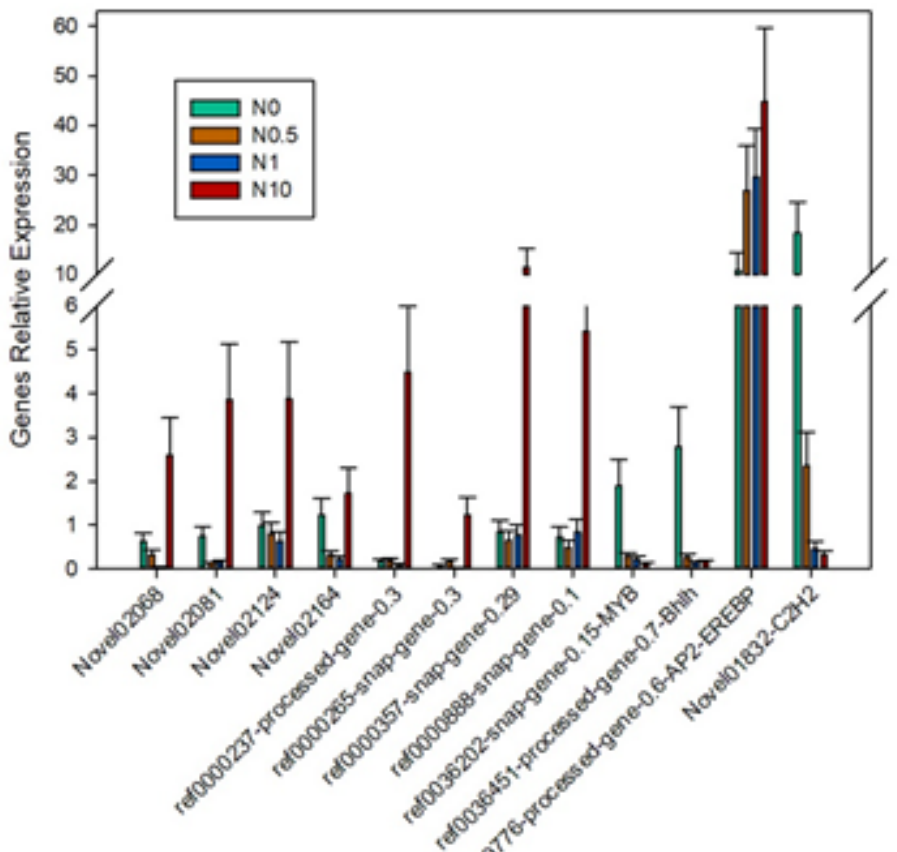

$\mathrm{D}$

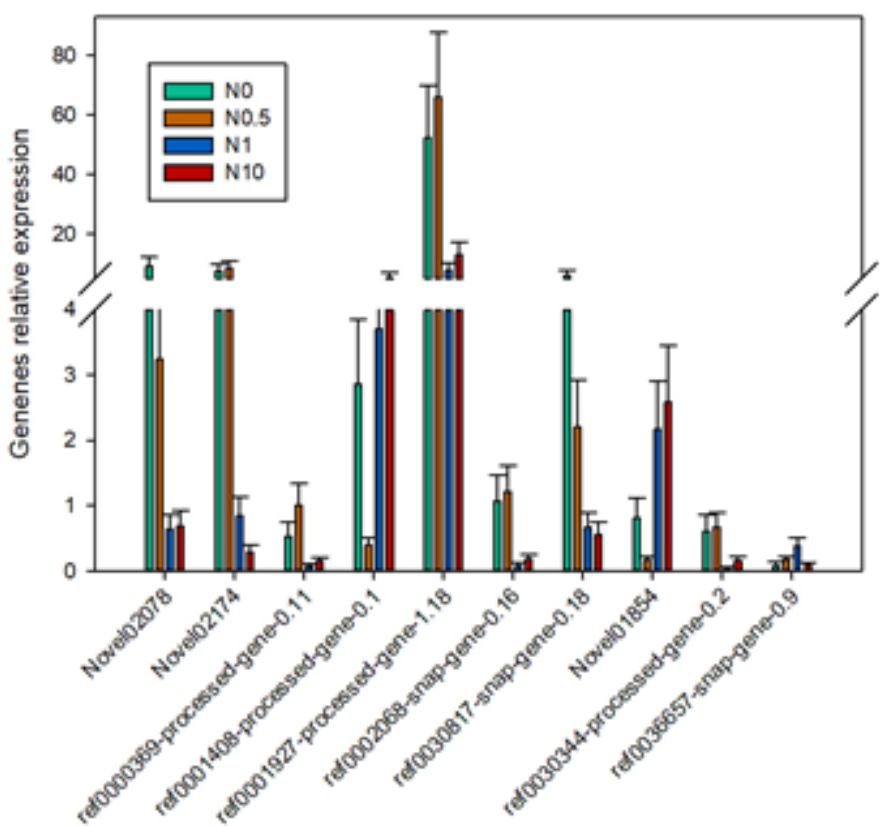

Figure 11

Expressions of genes selected for validation. $A$ and $C$ represents FPKM of genes related to nitrogen utilization and excessive nitrogen stress by RNA-Seq. B and D represents the relative expression of genes related to nitrogen utilization and excessive nitrogen stress by qRT-PCR.

\section{Supplementary Files}

This is a list of supplementary files associated with this preprint. Click to download. 
- tables1.xIsx

- tables4.xlsx

- tables3.xlsx

- tables2.xlsx 